\title{
HEATING AND IONIZATION OF THE
}

\author{
INTERSTELLAR MEDIUM
}

\author{
MICHAEL GREWING \\ Institut für Astrophysik und extraterrestrische Forschung der Universität Bonn und \\ Max-Planck-Institute für Radioastronomie, Bonn, F.R.G.
}

\begin{abstract}
Ionization and heating mechanisms are reviewed; some of them apply to the interstellar medium as a whole, others only to localized regions. Cooling processes are briefly summarized. Results are given from a recent model calculation for an intercloud medium heated by $\mathrm{X}$-rays.
\end{abstract}

\section{Introduction}

Over the past few years a large number of both observational and theoretical studies have been undertaken to reveal the physical state of the interstellar medium (ISM) and the processes that cause its ionization and heating. Observationally, new results have come from radioastronomical measurements, from optical studies, and - in the most spectacular form - from the OAO-C Copernicus satellite in the ultraviolet range. Theoretically, new processes that could cause an energy input into the interstellar gas have been studied, and further details of the so-called 'two-component model' of the ISM have been worked out. Also, the first successful attempts to study timedependent phenomena in interstellar space have been made.

As far as the theory is concerned, an excellent and very extensive review of the present state of the art has recently been given by Dalgarno and McCray (1972) so that in our discussion of the heating and ionization of the interstellar medium we can restrict ourselves to a broad outline of the relevant physical processes the details of which may be found in their paper. We shall start out by a discussion of various heating mechanisms. This is followed by a brief summary of cooling processes, and finally, we shall present results from a new model calculation for an X-ray heated intercloud medium in which an attempt was made to account for the observations from the Copernicus satellite.

\section{Heating Mechanisms}

The heating processes so far discussed in the literature may be divided into three categories depending on whether they are assumed to operate (a) continuously in space and time, (b) continuously in time but only in localized regions in space, or (c) discontinuously in both space and time.

(a) PROCESSES OPERATING CONTINUOUSLY IN SPACE AND TIME

A large variety of heating mechanisms have been suggested that fall in this category. Among these are the heating due to the light of the stars at $\lambda>912 \AA$, low energy 
cosmic rays (1-10 MeV) and soft X-rays $(\sim 100-300 \mathrm{eV})$, as well as the heating due to cloud collisions, hydromagnetic waves and the dissipation of the turbulent energy of the ISM. In our further discussion we shall concentrate upon the first three of these processes.

Basic to these heating mechanisms is the conversion of energy originally stored in some background radiation field (including the cosmic rays) into kinetic energy which, carried away e.g. by electrons, will then be thermalized in elastic collisions. The primary interaction process may be denoted as

$$
A+\left\{\begin{array}{c}
h v \\
p
\end{array}\right\} \rightarrow A^{+}+e^{-}+\left\{\begin{array}{c}
- \\
p^{\prime}
\end{array}\right\}
$$

where $A$ is an atom or ion that is transferred into its next higher stage of ionisation. Energy conservation determines the amount of kinetic energy carried away by the liberated electron $E_{\mathrm{e}}$. Heating occurs if

$$
E_{\mathrm{e}}>\left\langle E_{\mathrm{e}}\right\rangle
$$

where $\left\langle E_{\mathrm{e}}\right\rangle$ is the mean kinetic energy of the gas, determined by its temperature. For $10 \leqslant T \leqslant 10^{4} \mathrm{~K}, 8.6 \times 10^{-4} \leqslant\left\langle E_{\mathrm{e}}\right\rangle \leqslant 0.86 \mathrm{eV}$.

(i) Starlight at $\lambda>912 \AA$

If all Lyman-continuum photons are indeed trapped in $\mathrm{H}_{\mathrm{II}}$ regions around the stars producing them, the stellar radiation field terminates at the Lyman limit. It can therefore ionize only trace elements like $\mathrm{C}, \mathrm{Mg}, \mathrm{Si}, \mathrm{S}$, and $\mathrm{Fe}$ which are transferred to their first stage of ionization. In this process the liberated electrons obtain an average energy of roughly $2 \mathrm{eV}$ which is rapidly thermalized. However, due to the fact that this interaction process relies on the abundance of the trace elements the total heat input into the gas is small compared to what is required.

(ii) Low-Energy Cosmic Rays

The inadequacy of the stellar radiation field to explain the observed interstellar temperatures led Hayakawa (1960) to propose that low-energy cosmic rays might be a more favourable heating source. The primary ionization rate of neutral hydrogen in a collision with a fast proton is given by

$$
\zeta_{\mathrm{H}}=\int_{E_{0}}^{\infty} J(E) \sigma(E) \mathrm{d} E
$$

where $J(E)$ denotes the flux of cosmic ray protons and $\sigma(E)$ their interaction cross section. Since $J(E) \propto E^{-2.6}$ and $\sigma(E) \propto E^{-1}$, roughly, the value of the above integral is determined by the flux of the least energetic particles with energy $E_{0}$ that are still available in sufficient numbers. This energy $E_{0}$ depends on the mean free path of cosmic rays in the Galaxy which for a gas of unit density is approximately 530, 57, 
and $0.7 \mathrm{pc}$ for 3,1 , and $0.1 \mathrm{MeV}$ particles. These numbers suggest the choice $E_{0}=2$ $\mathrm{MeV}$, the value most often quoted in the literature.

In an ionization of hydrogen by a $2-\mathrm{MeV}$ proton, the liberated electron will carry away an average energy of $32 \mathrm{eV}$. Only part of this energy is available for heating, typically $5 \mathrm{eV}$, whereas the rest goes into secondary ionizations and excitations. The exact amount that is thermalized depends on the degree of ionization of the medium as shown e.g. by Dalgarno and McCray (loc. cit., Figure 7).

Despite the fact that theories assuming the heating of the ISM to be due to lowenergy cosmic rays have been worked out to various degrees of sophistication (see, e.g., Spitzer and Scott, 1969; Habing and Goldsmith, 1971; Bergeron and Souffrin, 1971; Jura and Dalgarno, 1972; Vogel, 1972 and references quoted therein), they are all subject to a major uncertainty which results from the difficulty of determining the flux of 2-MeV particles applicable to the ISM. These particles strongly interact with the solar wind, being scattered back and forth between solar wind magnetic field inhomogeneities, so that by the time they reach the Earth their spectral energy distribution is completely modified, even during periods of minimum solar activity. Earlier attempts to demodulate the observations have e.g. been made by Gloeckler and Jokipii (1967) and Webber (1968) (see also Silk and Steigman, 1970). However, as demonstrated by Urch and Gleeson (1972) measurements made near the Earth are indeed very badly suited for reconstructing the interstellar flux of low energy cosmic rays. Their results are shown in Figure 1.

As shown in this figure, Urch and Gleeson calculated the energy distribution resulting at the Earth from three vastly different input spectra, taking into account adiabatic expansion effects which had previously been overlooked. These results demonstrate that reliable estimates of the 2-MeV cosmic-ray flux in interstellar space will have to await measurements made at much larger distances from the Sun which can be carried out from space probes flying to the outer planets.

With actual measurements still to come, indirect arguments have been used to determine the hydrogen ionization rate due to $2-\mathrm{MeV}$ protons as $\zeta_{\mathrm{H}}=4 \times 10^{-16} \mathrm{~s}^{-1}$ (e.g., Field et al., 1969). This requires an energy density of $2-\mathrm{MeV}$ particles of $6 \times 10^{-14}$ erg $\mathrm{cm}^{-3}$, corresponding to a flux of roughly 0.2 particles $\mathrm{cm}^{-2} \mathrm{~s}^{-1}$ per one $\mathrm{MeV}$ energy range, which is very large on the basis of present observations.

(iii) Soft $X$-Rays

In view of the doubts that remain whether low-energy cosmic rays are sufficiently numerous to keep the interstellar gas heated and ionized, Silk and Werner (1969) suggested the heating and ionization by soft $\mathrm{X}$-rays as an alternative process. The primary ionization rate is given by

$$
\zeta=\int_{E_{0}}^{\infty} J(E) \sigma(E) \mathrm{d} E,
$$

where $J(E)$ denotes the flux of X-ray photons, $\sigma(E)$ the photoionization cross-section 


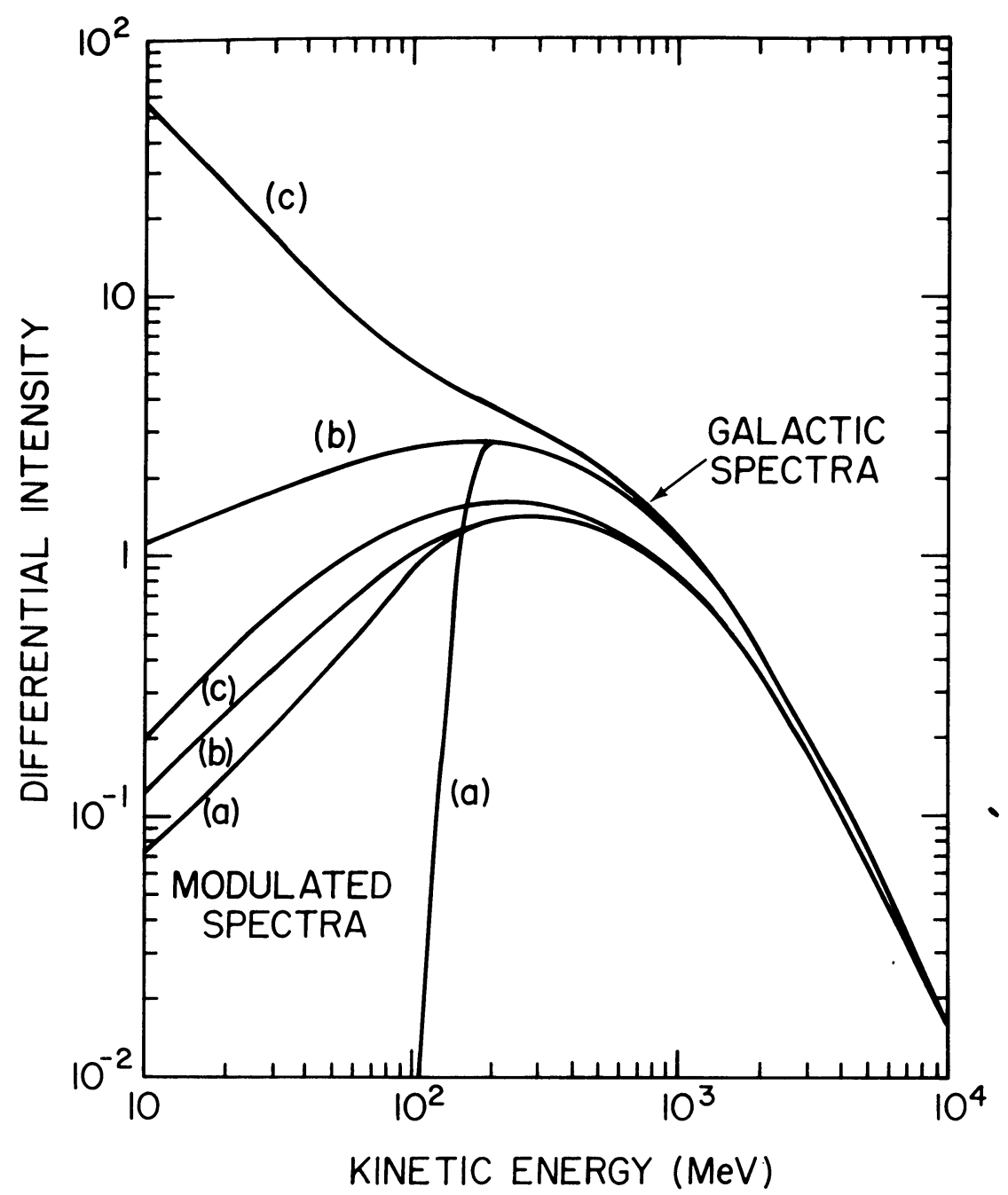

Fig. 1. Urch and Gleeson (1972) calculated the modulation effects that occur as low-energy cosmic-ray protons penetrate into the solar system where they are scattered back and forth between solar wind magnetic field inhomogeneities. Due to adiabatic expansion effects the cosmic-ray protons are degraded in energy and their spectral energy distribution is completely modified when the particles finally reach the Earth. This is shown here for three different (assumed) galactic spectra $(a, b, c)$ which would hardly be distinguishable by measurements from near the Earth.

(including inner shell contributions, Auger- and Coster-Kronig reactions), and $E_{0}$ the ionization threshold energy. Since $J(E)$ falls off with increasing energy, and since $\sigma(E) \sim E^{-3}$, roughly, the softest X-ray photons still available in sufficient numbers determine the ionization rate. The best information on the low-energy photon flux comes probably from the recent experiment by Yentis et al. (1972), which covers the 0.1-0.28-keV range. Results based on their data which are shown in Figure 2 will be presented in Section IV. 


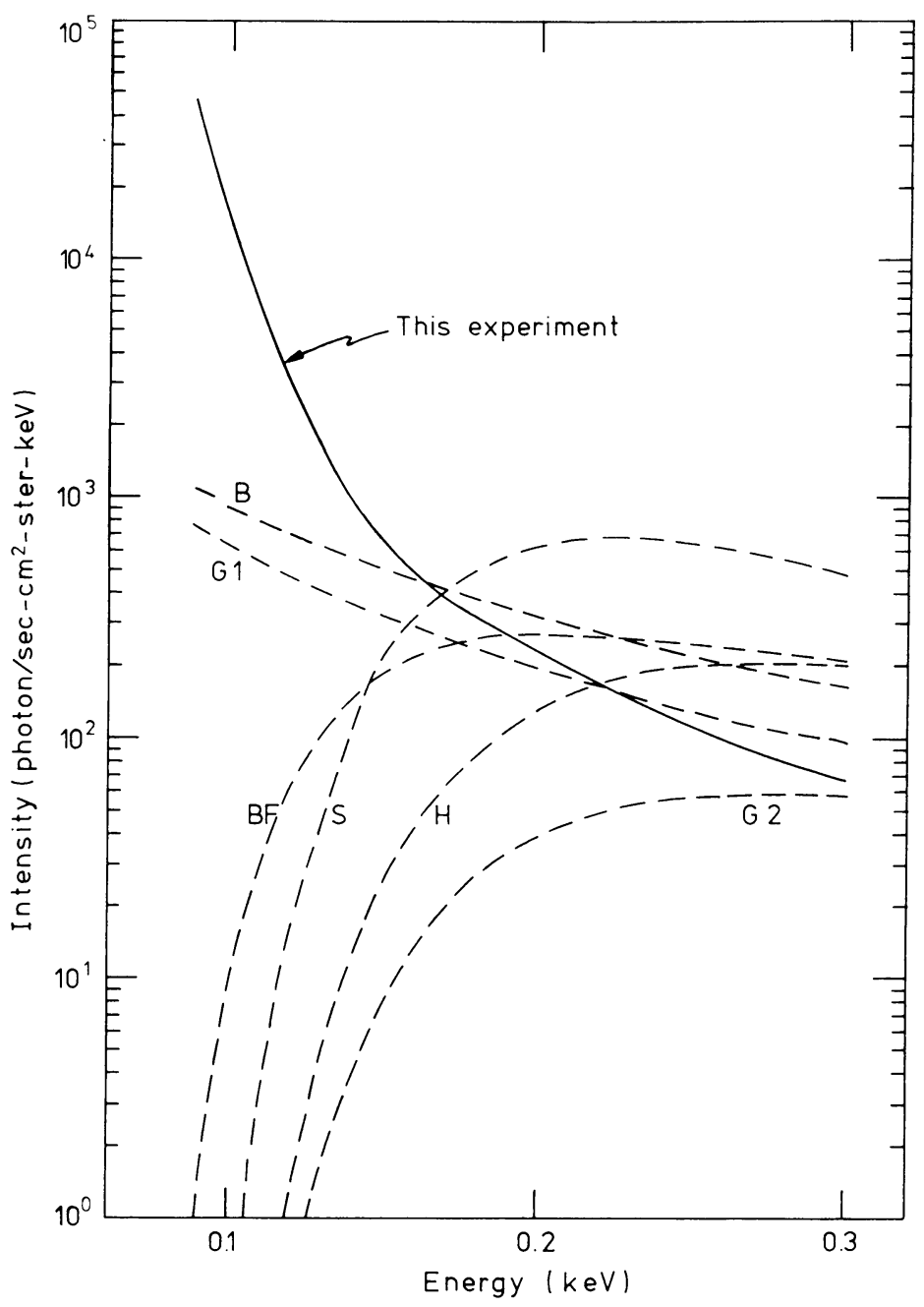

Fig. 2. This figure is taken from the paper of Yentis et al. (1972) who succeeded in extending the measurements of the soft $\mathrm{X}$-ray background to the $100-200 \mathrm{eV}$ range in a rocket experiment.

As shown in Figure 2, the observed flux of soft X-rays increases strongly towards lower energies, making the results rather crucially dependent on the choice of $E_{0}$. The mean free path of soft X-rays in the Galaxy is approximately 130, 9, and $1 \mathrm{pc}$ for 250,100 , and $50-\mathrm{eV}$ photons, assuming a gas density of 1 particle $\mathrm{cm}^{-3}$. These numbers suggest that for $n<1$, i.e. in the intercloud gas, $50 \leqslant E_{0} \leqslant 100 \mathrm{eV}$, whereas for $n>1$, i.e. for interstellar clouds, $E_{0}>100 \mathrm{eV}$ seems more appropriate.

(b) PROCESSES OPERATING CONTINUOUSLY IN TIME, BUT ONLY IN LOCALIZED REGIONS IN SPACE

Among the processes that fall into this category are the ionization and heating due 
to photons with $\lambda<912 \AA$, which originate (i) from O- and early-type B stars, (ii) from UV-stars, and (iii) from He-recombination processes in the case of an X-ray heated ISM. These mechanisms are particularly relevant for the intercloud regions, whereas the ones listed next pertain to interstellar clouds: (iv) heating due to photoelectron emission from irradiated grains, (v) collisional de-excitation of vibrational levels of $\mathrm{H}_{2}$ after photo-excitation, and (vi) photodestruction of $\mathrm{H}_{2}$-molecules.

(i) $O$ - and Early-Type B-Stars

Most obvious of course is the severe effect that Lyman-continuum photons generated by $\mathrm{O}$ - and early-type B-stars have on the nearby interstellar space in which they produce an $\mathrm{H}$ il region.

The enhanced matter density in regions where new stars form would normally guarantee that these $\mathrm{H}$ II regions are ionization-bounded. However, in particular Bstars may be sufficiently old to have moved into more normal regions of interstellar space where the densities are considerably lower so that much larger volumes can be ionized. This possibility was considered by Prentice and ter Haar (1969), Grewing and Walmsley (1971), Walmsley and Grewing (1971), and Richstone and Davidson (1972). Though their results are somewhat discrepant due to differences in the assumptions made, these authors show that O- and early-type B-stars play a substantial role for the ionization of the intercloud medium.

(ii) UV-Stars

A potentially very powerful further source of Lyman-continuum quanta are the socalled UV-stars (Hills, 1972). Model calculations predict that on their way from the red giant to the white dwarf stage stars will have surface temperatures of $T_{\text {eff }} \gtrsim 10^{5} \mathrm{~K}$ for times $t \sim 10^{6} \mathrm{yr}$. Their luminosity would be $L \sim 10^{2} L_{\odot}$. The source of this energy is the gravitational contraction of the star, and the numbers given depend rather crucially on what amount of the energy liberated is lost, e.g. in neutrinos. This question is still open, and no UV-star has - to my knowledge - as yet been seen, contrary to what one would expect on the basis of the number density estimate given by Hills (loc. cit.). However, if one uses the numbers adopted by Hills, all the intercloud matter in the Galaxy could be ionized by these stars.

\section{(iii) EUV-Photons from Helium-Recombinations}

In those regions of interstellar space which are heated and ionized by soft X-rays, recombinations of $\mathrm{He} \mathrm{II}$ and $\mathrm{He}$ III lead to a further production of photons in the Lyman-continuum band. As shown in Section IV, these photons play an essential role in the ionization of hydrogen in such regions and add a little to the heating.

\section{(iv) Photoelectron Emission from Interstellar Grains}

Watson (1972) first noted that for likely interstellar grain materials except ice, photoemission of electrons is expected from laboratory data to be a relatively efficient process for photon energies of $\sim 10-13.6 \mathrm{eV}$. The average energy of the ejected photo- 
electron depends somewhat on the grain material and on the photon energy, but will typically be $2 \mathrm{eV}$, well in excess of the mean kinetic energy in interstellar clouds. Using recent determinations of the galactic ultraviolet flux, Watson arrives at the conclusion that the energy input into $\mathrm{H}$ I clouds due to this mechanism is comparable to that from proposed fluxes of low energy cosmic rays.

\section{(v) Collisional De-Excitation of Vibrational Levels of $\mathrm{H}_{2}$}

After photo-excitation of molecular hydrogen in the Lyman and Werner bands, in the majority of cases the molecules return to the ground electronic state in a vibrationally excited level. As pointed out by Stecher and Williams (1973) collisional deexcitation of these levels will provide a heating mechanism, which generally however is small compared to the one to be discussed next.

\section{(vi) Radiative Dissociation of $\mathrm{H}_{2}$}

As stated above in the majority of all cases $(\sim 73 \%)$ the excitation of molecular hydrogen by UV-photons will lead to vibrationally excited molecules in their electronic ground state. In the remaining cases $(\sim 27 \%)$, however, the molecules cascade into the vibrational continuum of the ground state whereby they dissociate. The pair of $\mathrm{H}$-atoms that comes out of this photo-destruction mechanism will carry an average kinetic energy of $\sim 0.5 \mathrm{eV}$ according to an estimate by Dalgarno, which exceeds the mean kinetic energy in interstellar clouds. Milgrom et al. (1973) pointed out the importance of this heating mechanism and compared it to the heating due to the photoeffect on grains.

We should emphasize that the processes (iv), (v), and (vi) depend critically on the penetration of photons with energies $10-13.6 \mathrm{eV}$ into the clouds. Along with the molecules and the grains, carbon will be competing for these photons. For clouds of sufficient optical thickness one will have to distinguish between a $\mathrm{C}_{\mathrm{I}}$-core and an $\mathrm{C}$ II-envelope in which photons with energies $10-11.2 \mathrm{eV}$ will all be absorbed. A first attempt to take these effects into account has been made by Walmsley (1973).

\section{(c) TIME-DEPENDENT PROCESSES IN LOCALIZED REGIONS IN SPACE}

As a third category we shall briefly consider time-dependent heating mechanisms that apply to localized regions in space. It was only fairly recently that the potential importance of short-term large-scale energy releases connected with gravitational collapse as it occurs e.g. during a supernova explosion for the heating and ionization of the ISM was noted. In their analysis of data pertaining to the Gum nebula, Brandt et al. (1971) were led to the hypothesis that this region in space is a fossil $\mathrm{H}$ II region generated by a SN-flash some $10^{4} \mathrm{yr}$ ago when the Vela pulsar was formed. Though the model they suggest for the Gum nebula is still open to discussion, it served to trigger a rapid series of theoretical studies of time-dependent models for the ISM (e.g., Schwarz, 1973; Kafatos, 1973; Gerola et al., 1973).

The most recent discussion of the possible role of supernovae for the ionization and heating of the ISM was given by Cox (1973). From it one may conclude that 
SN provide so much energy (in the form of UV quanta, cosmic rays and shockwaves) that their influence on the ISM will indeed not be limited to localized regions in space but that instead $\mathrm{SN}$ rather than the X-ray background or the cosmic ray background provide the fundamental source of energy at least for the intercloud medium in our Galaxy.

\section{Cooling Mechanisms}

An excellent and very detailed review of the cooling processes operating in interstellar space has been given in the paper by Dalgarno and McCray (1972). We shall therefore be very brief here.

Basic to all cooling mechanisms is the conversion of kinetic energy by inelastic collisions into line radiation. Except in dense interstellar clouds where one has to solve a radiation transfer problem, the ISM may generally be assumed to be optically thin at the relevant wavelengths. The photons produced can thus freely escape, causing a loss of energy to the system.

The cooling processes so far considered in the literature may be grouped into three main classes depending on whether they are based on collisions involving free electrons, $\mathrm{H}$-atoms, or $\mathrm{H}_{2}$-molecules. Most of the mechanisms belonging to the second class have already been considered in the classical papers by Spitzer $(1948,1949)$. He concentrated on these processes since at that time free electrons were thought to be extremely rare in interstellar space $\left(\sim 10^{-4} \mathrm{~cm}^{-3}\right)$. The mechanisms that fall into this group are

$$
L_{\mathrm{Hi}}, L_{\mathrm{Ha}}, L_{\mathrm{Hm}}, L_{\mathrm{Hg}}
$$

denoting the cooling due to hydrogen-ion and hydrogen-atom collisions in which ground-state fine-structure transitions are excited, the cooling produced by the collisional excitation of rotational levels of molecules, and the cooling due to collisions of hydrogen atoms with grains. For $L_{\mathrm{Hi}}$ the cases $i=\mathrm{C} \mathrm{II}, \mathrm{Si}$ II and Fe II are particularly important. For $L_{\mathrm{Ha}}$ the cases $a=\mathrm{O}$ I and $\mathrm{C}_{\mathrm{I}}$ have been considered. For $L_{\mathrm{Hm}}$ the cases $m=\mathrm{H}_{2}, \mathrm{HD}, \mathrm{CO}, \mathrm{CN}$, and $\mathrm{CH}$ have been discussed in the literature. A compilation of all these processes and detailed references to rate estimates etc. may be found in Dalgarno and McCray's paper (loc. cit.).

In their paper Dalgrano and McCray also discuss the processes

$$
L_{\mathbf{H}_{2} i}, L_{\mathbf{H}_{2} a}, L_{\mathbf{H}_{2} m},
$$

which denote the excitation of ions, atoms, and molecules in collisions with $\mathrm{H}_{2}$ molecules. These processes may be relevant in interstellar clouds where the $\mathrm{H}_{2}$ abundance is known to be high from observations of the Copernicus satellite (Spitzer et al., 1973). The cases so far considered are $i=\mathrm{CII}, a=\mathrm{O}_{\mathrm{I}}, \mathrm{CI}$, and $m=\mathrm{H}_{2}$, and $\mathrm{H}_{2} \mathrm{CO}$.

The processes that belong to the first class mentioned may be denoted as

$$
L_{\mathrm{e} i}, L_{\mathrm{e} a}, L_{\mathrm{e} m} .
$$

In electron-ion collisions the excitation of ground-state fine-structure levels dominates 
at lower temperatures $(i=\mathrm{C} \mathrm{II}, \mathrm{Si} \mathrm{II}$, and $\mathrm{Fe} \mathrm{II})$ whereas at intermediate temperatures $(600-6000 \mathrm{~K})$ the excitation of metastable levels is most important ( $i=\mathrm{C} \mathrm{II}$, O II, $\mathrm{Si}$ II, S II, and Fe II). At higher temperatures the collisional excitation of allowed dipole transitions of atoms $(a=\mathrm{H} \mathrm{I}, \mathrm{O} \mathrm{I}$, and $\mathrm{NI})$ must be taken into account. In their paper Dalgarno and McCray give a very detailed summary of all these processes as well as of the $L_{\mathrm{e} m}$ collisions where the cases $m=\mathrm{CO}, \mathrm{CN}, \mathrm{CH}$, and $\mathrm{CH}^{+}$are considered.

\section{Results from Recent Model Calculations for an X-Ray Heated Intercloud Medium}

Recent advances in measuring the soft X-ray background in the $100-280 \mathrm{eV}$ range (Yentis et al., 1972), improved photo-ionization cross-sections (Barfield et al., 1972), and a wealth of detailed observational data from the Copernicus satellite (Rogerson

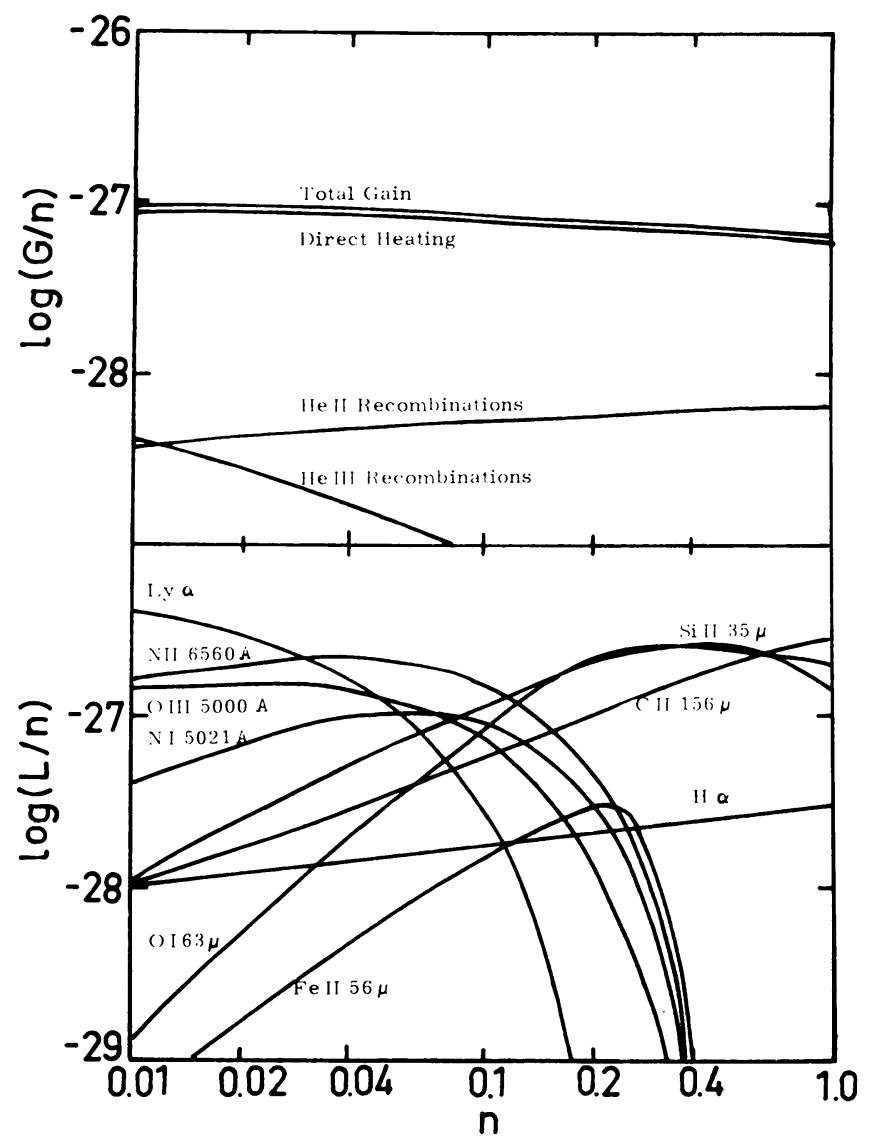

Fig. 3. This as well as the following two figures are taken from the paper by Grewing and Walmsley (1974) in which results are given from model calculations for an X-ray heated intercloud medium. In the top part of this figure the energy gain of the gas is shown that results from the observed X-ray background shown in Figure 2. In the bottom part of the figure the energy loss of the gas due to various line transitions is plotted. 
et al., 1973; Morton et al., 1973) made it seem worthwhile to re-compute the ionization and thermal balance of an X-ray heated intercloud medium. A full description of this new model is given by Grewing and Walmsley (1974) so that we can restrict ourselves to a few main points.

Adopting the X-ray spectrum as measured by Yentis et al. (loc. cit.) the primary ionization rate for hydrogen is found to be $3.4 \times 10^{-17} \mathrm{~s}^{-1}$. Primary ionization rates have also been calculated for 47 further atoms or ions, using the photo-ionization cross-sections of Barfield et al. (loc. cit.). Auger- and Coster-Kronig contributions have been considered but appear to be negligible except for the ions Al II, Si II, and Fe II. Large effects, however, are found to come from secondary ionization processes. For hydrogen, where they are largest, we obtain a ratio of secondary to primary ionization rates of 3.1 and 5.1 for a density of 0.1 and $1 \mathrm{~cm}^{-3}$, respectively. Indeed, even the rate of hydrogen ionizations following He-recombinations is found to exceed the primary rate by factors 1.45 and 1.53 at these same densities.

The total heat input into the gas from the observed X-ray background is shown in the top part of Figure 3. The energy gain per particle is rather constant with density, and is for all densities essentially determined by the direct heating whereas the heating that results from $\mathrm{He}$ recombinations is only of secondary importance.

In calculating the thermal equilibrium of the gas, cooling rates were used which differ from that of previous authors (e.g. Bergeron and Souffrin, 1971; Habing and Goldsmith, 1971) in two respects. Firstly, the effects of the ionization balance were

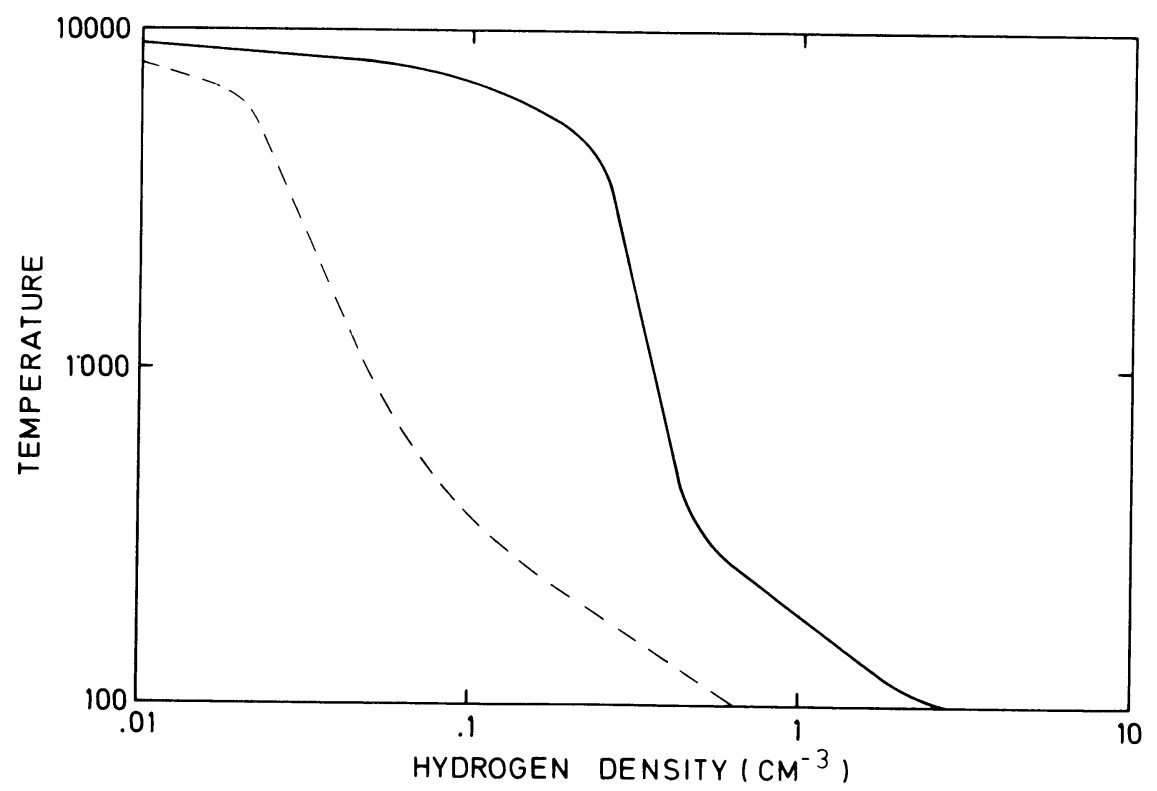

Fig. 4. By solving the thermal balance equation the equilibrium temperature of the gas was determined as a function of the gas density. This result depends strongly on the assumed abundances of heavy elements. The full line shows the temperature that is obtained using abundances determined by the OAO-C satellite (see text), whereas the dashed line is based on solar abundances. 
taken into account, and secondly, heavy element abundances as determined by the OAO-C satellite for the line of sight towards the star $\alpha$ Leo were used (Rogerson et al., loc. cit.). The cooling losses that then result are shown in the bottom part of Figure 3.

In Figure 4 the equilibrium temperature is plotted as a function of the hydrogen density. The full line corresponds to the OAO-C abundances. It differs significantly from the temperature derived for normal solar abundances shown as a dashed curve. It is particularly interesting that the X-ray flux determined by Yentis et al. (loc. cit.) combined with the abundance determinations from the Copernicus satellite allows the maintenance of a gas density of $0.2 \mathrm{~cm}^{-3}$ at a temperature of $5000 \mathrm{~K}$. These values

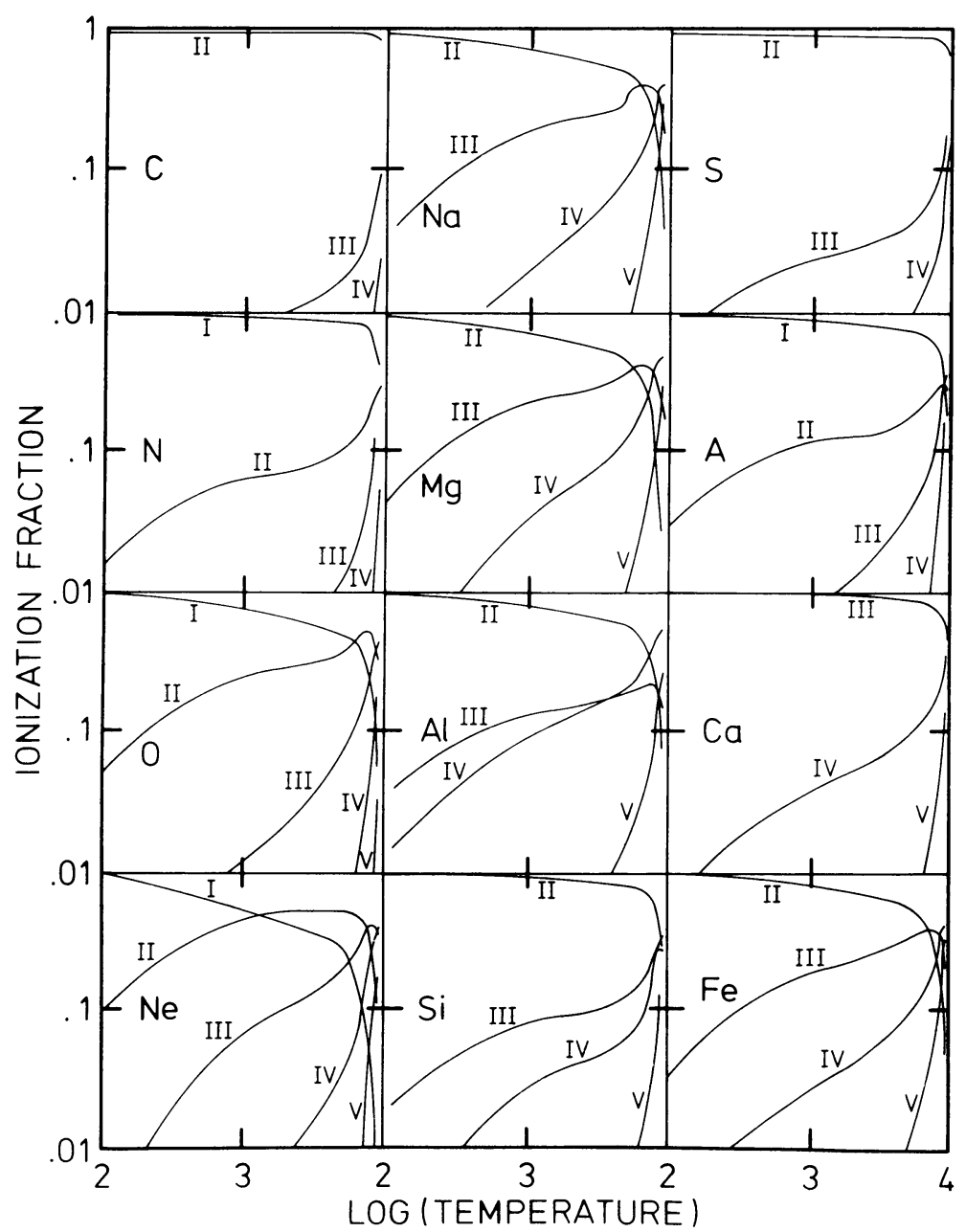

Fig. 5. The fractional ionization of the elements $\mathrm{C}, \mathrm{N}, \mathrm{O}, \mathrm{Ne}, \mathrm{Na}, \mathrm{Mg}, \mathrm{Al}, \mathrm{Si}, \mathrm{S}, \mathrm{A}, \mathrm{Ca}$, and Fe is shown as a function of the equilibrium temperature (see text for further details of the calculations). 
agree well with those derived from an analysis of 21-cm hydrogen line profiles (e.g., Mebold, 1972).

In Figure 5 the fractional ionization of the elements $\mathrm{C}, \mathrm{N}, \mathrm{O}, \mathrm{Ne}, \mathrm{Na}, \mathrm{Mg}, \mathrm{Al}, \mathrm{Si}$, $\mathrm{S}, \mathrm{A}, \mathrm{Ca}$, and $\mathrm{Fe}$ is shown as a function of the equilibrium temperature $T$ for the OAO-C abundances. These results should serve as a test whether the intercloud $\mathrm{HI}_{\mathrm{I}}$ gas is indeed ionized by X-rays.

\section{Conclusions}

Over the past few years our understanding of many individual physical processes that may be relevant in interstellar space has greatly increased. In particular, the 'two-component model' of the ISM has been worked out in great detail, and considerable progress has also been made on the front of time-dependent models. It remains doubtful, however, whether this has really helped us to derive from the observations a quantitatively more reliable picture of the actual conditions existing in interstellar space. By looking at a distant star our line of sight might intersect interstellar clouds, intercloud matter heated by cosmic rays, X-rays or UV quanta, a tenuous $\mathrm{H}$ II region around an early-type B-star, a normal $\mathrm{H}$ II region, a fossil Strömgren sphere and even the supernova-produced tunnel-network proposed recently by Cox (1973). In our measurement we are integrating over physically very different regimes. In order to interpret such observations in a quantitative manner we would therefore firstly have to really understand what is going on in each of the different regions that can be located along a given line of sight, and secondly, we would have to find methods to recognize their actual contributions to what we see.

\section{References}

Barfield, W. D., Koontz, G. D., and Huebner, W. F.: 1972, J. Quant. Spectr. Radiative Transfer 12, 1409. Bergeron, J. and Souffrin, S.: 1971, Astron. Astrophys. 11, 40.

Brandt, J. C., Stecher, T. P., Crawford, D. I., and Maran, S. P.: 1971, Astrophys. J. Letters 163, L99.

Cox, D.: 1973, Report given at the IAU General Assembly, Sydney, Australia.

Dalgarno, A. and McCray, R. A.: 1972, Ann. Rev. Astron. Astrophys. 10, 375.

Field, G. B., Goldsmith, D. W., and Habing, H. J. : 1969, Astrophys. J. Letters 155, L149.

Gerola, H., Iglesias, E., and Gamba, Z.: 1973, Astron. Astrophys. 24, 369.

Gloeckler, G. and Jokipii, J. R.: 1967, Astrophys. J. Letters 148, L41.

Grewing, M. and Walmsley, C. M.: 1971, Astron. Astrophys. 11, 65.

Grewing, M. and Walmsley, C. M.: 1974, Astron. Astrophys. 30, 281.

Habing, H. J. and Goldsmith, D. W.: 1971, Astrophys. J. 166, 525.

Hayakawa, S.: 1960, Publ. Astron. Soc. Japan 12, 110.

Hills, J. G.: 1972, Astron. Astrophys. 17, 155.

Jura, M. and Dalgarno, A.: 1972, Astrophys. J. 174, 365.

Kafatos, M.: 1973, Astrophys. J. 182, 433.

Mebold, U.: 1972, Astron. Astrophys. 19, 13.

Milgrom, M., Panagia, N., and Salpeter, E. E.: 1973, Astrophys. Letters 14, 73.

Morton, D. C., Drake, J. F., Jenkins, E. B., Rogerson, J. B., Spitzer, L., and York, D. G.: 1973, Astrophys. J. Letters 181, L103.

Prentice, A. J. R. and ter Haar, D.: 1969, Monthly Notices Roy. Astron. Soc. 146, 423.

Richstone, D. O. and Davidson, K.: 1972, Astron. J. 77, 298. 
Rogerson, J. B., York, D. G., Drake, J. F., Jenkins, E. B., and Spitzer, L.: 1973, Astrophys. J. Letters 181, L110.

Schwarz, J.: 1973, Astrophys. J. 182, 449.

Silk, J. and Steigman, G.: 1970, Astrophys. Space Sci. 9, 304.

Silk, J. and Werner, M.: 1969, Astrophys. J. 158, 185.

Spitzer, L.: 1948, Astrophys. J. 107, 6.

Spitzer, L.: 1949, Astrophys. J. 109, 337.

Spitzer, L. and Scott, E. M.: 1969, Astrophys. J. 158, 161.

Spitzer, L., Drake, J. F., Jenkins, E. B., Morton, D. C., Rogerson, J. B., and York, D. G. : 1973, Astrophys. J. 181, L116.

Stecher, T. P. and Williams, D. A.: 1973, Monthly Notices Roy. Astron. Soc. 161, 305.

Urch, I. H. and Gleeson, L. J.: 1972, Astrophys. Space Sci. 16, 55.

Vogel, U.: 1971, Thesis, University of Bonn.

Walmsley, C. M.: 1973, Astron. Astrophys. 25, 129.

Walmsley, C. M. and Grewing, M.: 1971, Astrophys. Letters 9, 185.

Watson, W. D.: 1972, Astrophys. J. 176, 103.

Webber, W. R.: 1968, Australian J. Phys. 21, 845.

Yentis, D. J., Novick, R., and van den Bout, P.: 1972, Astrophys. J. 177, 365.

Michael Grewing

Institut für Astrophysik und extraterr. Forschung der Universität Bonn,

Auf dem Hügel 71,

5300 Bonn, F.R.G. 